\title{
Crystal structure of potassium hexabismuth enneaoxide iodide, $\mathrm{KBi}_{6} \mathrm{O}_{9} \mathrm{I}$
}

\author{
U. Eggenweiler, E. Keller and V. Krämer
}

Albert-Ludwigs-Universität Freiburg, Kristallographisches Institut, Hebelstr. 25, D-79104 Freiburg, Germany

Received August 13, 1996, transferred to 2nd update of database ICSD in 1997, CSD-No. 402605

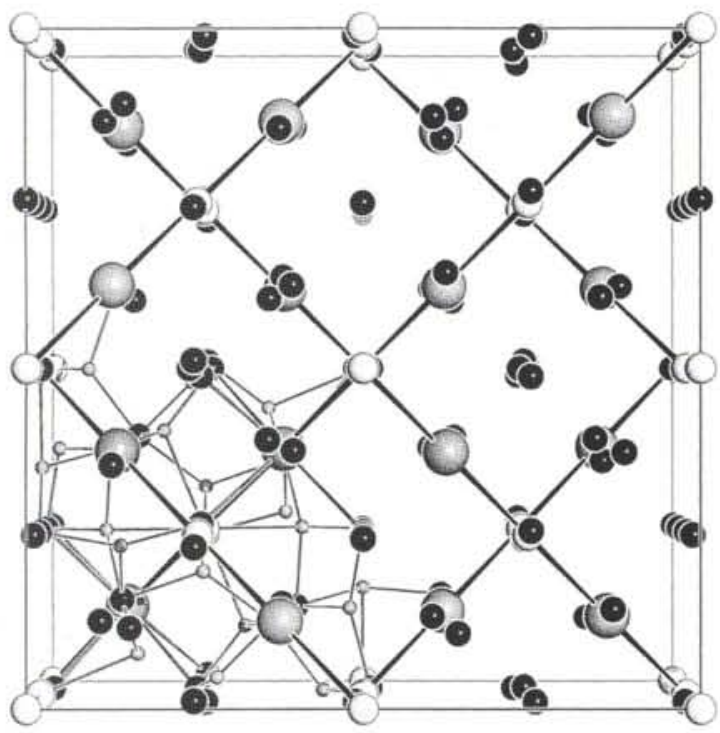

Fig. 1. Oxygen atoms with $x, y, z>0.5$ have been omitted for sake of clarity.
Source of material: Small single crystals of $\mathrm{KBi}_{6} \mathrm{O} 9 \mathrm{I}$ grew after drop by drop addition of a $0.005 n$ solution of $\mathrm{BiI}_{3}$ in $3 \mathrm{n} \mathrm{HI}$ to $10 \mathrm{n} \mathrm{KOH}$.

The $\mathrm{K}, \mathrm{Bi}$ and $\mathrm{I}$ atoms form a distorted bcc-like substructure. The $\mathrm{K}$, I substructure consists of non-intersecting infinite linear chains parallel to $<111>$. The coordination polyhedra of $\mathrm{Bi}$ are similar to those ones found in $\mathrm{\beta}-\mathrm{Bis} \mathrm{O} \mathrm{I}$ (see ref. 1).

Bi6IKO9, cubic, $I a \overline{3} d$ (No. 230), $a=17.254(3) \AA, V=5136.5 \AA^{3}$, $Z=16, R(F)=0.019, R_{\mathrm{w}}\left(F^{2}\right)=0.037$.

Table 1. Parameters used for the $\mathrm{X}$-ray data collection

Crystal:

yellow, distorted thomb-dodecahedron,

Wavelength:

$\mu$ : size $0.057 \times 0.116 \times 0.135 \mathrm{~mm}$

Mo $K_{\alpha}$ radiation $(0.71073 \AA)$

$806.50 \mathrm{~cm}^{-1}$

Diffractometer: $\quad$ Enraf-Nonius CAD4

Scan mode: $\quad \omega / 2 \theta$

Tmeasurement: $\quad 293 \mathrm{~K}$

$2 \theta_{\max }: \quad 56^{\circ}$

$\mathrm{N}(h \mathrm{kl})_{\text {unique: }} \quad 520$

Criterion for $I_{0}: \quad I_{0}>2 \sigma\left(I_{0}\right)$

$\mathrm{N}($ param) refined. $\quad 30$

Programs: $\quad$ SHELXS-86, SHELXL-93

Table 2. Final atomic coordinates and displacement parameters (in $\AA^{2}$ )

\begin{tabular}{|c|c|c|c|c|c|c|c|c|c|c|}
\hline Atom & Site & $x$ & $y$ & $z$ & $U_{11}$ & $U_{22}$ & $U_{33}$ & $U_{12}$ & $U_{13}$ & $U_{23}$ \\
\hline $\mathrm{Bi}(\mathrm{l})$ & $48 g$ & $-0.10671(2)$ & $7 / 8$ & $0.14329(2)$ & $0.0107(1)$ & $0.0132(2)$ & $U_{11}$ & $-0.0008(1)$ & $-0.0015(1)$ & $-U_{12}$ \\
\hline $\mathrm{Bi}(2)$ & $48 f$ & $0.01194(2)$ & 0 & $1 / 4$ & $0.0092(2)$ & $0.0092(2)$ & $0.0116(2)$ & 0 & 0 & $-0.0004(1)$ \\
\hline $\mathrm{K}(1)$ & $16 a$ & 0 & 0 & 0 & $0.0185(9)$ & $U_{11}$ & $U_{11}$ & $0.004(1)$ & $U_{12}$ & $U_{12}$ \\
\hline I(1) & $16 b$ & $1 / 8$ & $1 / 8$ & $1 / 8$ & $0.0177(3)$ & $U_{11}$ & $U_{11}$ & $0.0042(3)$ & $U_{12}$ & $U_{12}$ \\
\hline$O(1)$ & $96 h$ & $-0.0633(4)$ & $0.0008(3)$ & $0.1523(4)$ & $0.010(3)$ & $0.010(3)$ & $0.015(3)$ & $0.000(2)$ & $-0.003(2)$ & $-0.002(2)$ \\
\hline$O(2)$ & $48 g$ & $-0.0216(3)$ & $7 / 8$ & $0.2284(3)$ & $0.015(2)$ & $0.003(3)$ & $U_{11}$ & $-0.001(2)$ & $-0.008(3)$ & $-U_{12}$ \\
\hline
\end{tabular}

\section{References}

1. Ketterer, J.; Keller, E.; Krämer, V.: Crystal structure of bismuth oxide iodide $\beta$-BisO-II. Z. Kristallogr. 172 (1985) 63-70.

2. Sheldrick, G. M.: SHELXS-86. Program for the solution of crystal structures. University of Göttingen, Germany 1986.

3. Sheldrick, G. M.: SHELXL-93, a program for refining crystal structures. University of Göttingen, Germany 1993. 